\title{
Particle acceleration at quasi-perpendicular non-relativistic high Mach number shocks
}

\author{
Naveen Kumar* and Brian Reville \\ Max-Planck Institute for Nuclear Physics, \\ Saupfercheckweg 1, Heidelberg, Germany \\ E-mail: naveen. kumar@mpi-hd.mpg.de
}

Electron and ion acceleration at a non-relativistic collisionless shocks is studied by employing large scale one-dimensional particle-in-cell (PIC) simulations in the de-Hoffmann and Teller (dHT) frame of reference. We demonstrate that diffusive shock acceleration of both electrons and ions occurs in quasi-perpendicular shock configurations at large Alfvén Mach numbers. We also identify the role of precursor waves on the electron energization in the upstream region. The emergence of a significant non-thermal ion component holds important implications for observations of hadronic emission from collisionless shocks occurring for example in supernova remnants, and colliding stellar winds.

$37^{\text {th }}$ International Cosmic Ray Conference (ICRC 2021)

July 12 th - 23rd, 2021

Online - Berlin, Germany

\footnotetext{
${ }^{*}$ Presenter
} 


\section{Introduction}

Collisionless shocks are common in many astrophysical environments and believed to be responsible for accelerating cosmic rays to high energies, as inferred from non-thermal $\mathrm{x}$-ray and $\gamma$-ray measurements [1]. Supernova remnants (SNR) remain the most plausible source of Galactic cosmic rays (CRs) up to the knee [2]. Cosmic-ray acceleration at shocks is thought to proceed via diffusive shock acceleration (DSA), where the cosmic ray particles repeatedly cross the shock while being scattered on magnetic field inhomogeneities. Of particular interest are quasi-perpendicular shocks $\left(\theta>60^{\circ}\right)$, where $\theta$ is the angle between the magnetic field and the shock normal surface $[3,4]$. Oblique shocks are, in principle, fast accelerators and should be prevalent in various astrophysical scenarios. In general, if shock velocities and magnetic field directions are uncorrelated, it follows that half of the shock will have quasi-perpendicular orientation. It has been recently suggested that highly oblique shocks are disfavoured as accelerators of protons/ions while still accelerating electrons to high energies [5, 6].

Though, DSA provides a generic explanation for the power-law spectra of particle undergoing shock acceleration, the mass difference between electrons and ions necessitates different mechanisms to facilitate shock acceleration, or injection to the process. Sufficiently energetic ions, by virtue of their greater rest mass and hence a larger gyro-radius naturally see the shock, whose thickness is on the order of the gyro radius of the incoming ions as measured in the downstream magnetic field, as narrow discontinuity. However, for electrons to be efficiently accelerated by a shock, a pre-heating mechanism is required that can initiate the DSA process of this lighter species. This problem is known as the "injection problem". On a microphysical level, the electrons and ions accelerations are linked with each other. Thus, it is instructive to examine electron and ion acceleration at highly oblique shocks.

\section{PIC simulations}

For PIC simulations, we use the open source code SMILEI [7]. For initialising the system, we solve the Rankine-Hugoniot jump conditions to determine the downstream parameters (superscript $d$ ) that connect the upstream parameters (superscript $u$ ) [8]. We use the de-Hoffmann and Teller (dHT) frame of reference for our simulations [9, 13]. On making use of relations, $\beta_{e}^{u}=2\left(M_{A} / M_{S}\right)^{2}, \omega_{c e}^{u} / \omega_{p e}^{u}=\left(v_{1 x} / c\right)\left(m_{i} / m_{e}\right)^{1 / 2} / M_{A}$, and $v_{t h e}^{u} / c=\left(M_{A} / M_{S}\right)\left(\omega_{c e}^{u} / \omega_{p e}^{u}\right)$, one can easily estimate the downstream parameters. Here $c$ is the velocity of light in vacuum, $\omega_{p e}$ is the electronic plasma frequency of the upstream region, $M_{A}=63$ and $M_{S}=70$ are the Alfvénic and sonic Mach numbers, respectively. The upstream plasma has drift velocity $v_{1 x}=0.15 c$ and $\left(\omega_{c e}^{u} / \omega_{p e}^{u}\right)$ and $v_{t h e}^{u}$ denote the electron cyclotron to electron plasma frequency ratio and the electron thermal velocity, respectively. Furthermore, we assume $\beta_{e}^{u}=\beta_{i}^{u} \approx 1.8$, implying ion to electron temperature ratio $T_{i}^{u}=T_{e}^{u}$, and $T_{i}^{d} / T_{e}^{d}=4$ similar to previous studies on collisionless shocks [13, 14]. The oblique magnetic field, $\mathbf{B}=B_{x} \hat{x}+B_{z} \hat{z}$ makes an angle of $\theta=66^{\circ}$ with respect to the shock normal in the upstream direction. For the sake of computational efficiency we assume a reduced ion to electron mass ratio $m_{i} / m_{e}=100$, and grid and time resolutions of $d x=0.1 c / \omega_{p e}$ and $d t=0.095 \omega_{p e}^{-1}$, respectively. The shock thickness is of the order of the ion gyro radius $\left(\rho_{i}=m_{i} v_{1 x} / e B^{d}\right)$. We also use two groups of test particles, each group contains 1000 

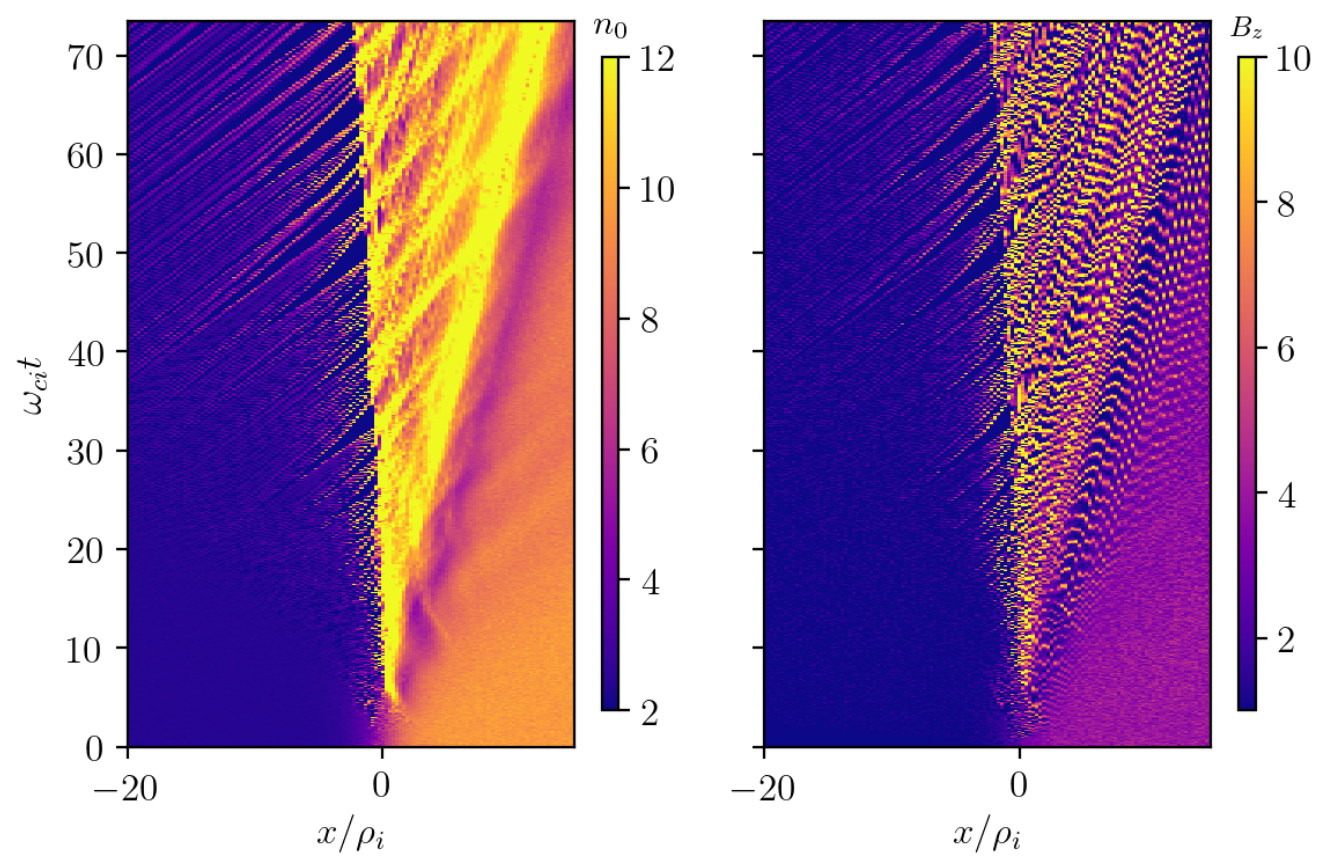

Figure 1: Zoomed-in view of the density map and the reformation of the transverse magnetic field. Time and the length are normalized to downstream ion gyro frequency and ion gyro radius respectively. Initial shock discontinuity is located at $x / \rho_{i}=0$.

particles (electrons and ions), in the upstream regions to understand the electron heating and ion acceleration mechanisms. These test particles have all other parameters identical to the upstream plasma. They experience the self-consistent plasma fields but do not participate in charge and current generation in the PIC simulations.

\subsection{Shock reformation}

Fig. 1 shows the evolution of the discontinuity, resulting in the formation of a well-defined regions associated with the shock, e.g. shock foot, shock jump and the overshoot regions. The magnetic field shows a similar pattern. One striking observation is the formation of spiky features both in the plasma density and the magnetic field maps. These features are a consequence of the shock reformation process intricately linked with the reflection of the incoming upstream ions at the shock front $[9,13]$. These features grow in time and affect the incoming plasma particles in the upstream region. There is onset of electrostatic and electromagnetic instabilities at the shock front $[10-12,15,16]$. These instabilities provide the requisite heating mechanism needed for the electron "injection problem".

\subsection{Particle spectra and trajectories}

Fig. 2 shows the spectra of electrons and ions on the downstream of the shock. One can immediately see the generation of non-thermal electrons (upper panel). The population of nonthermal ions are easier to visualise when one transforms back to the shock normal frame. This is due to boost in the $\hat{z}$-direction being of the order of the shock accelerated ions' velocity. The ion 

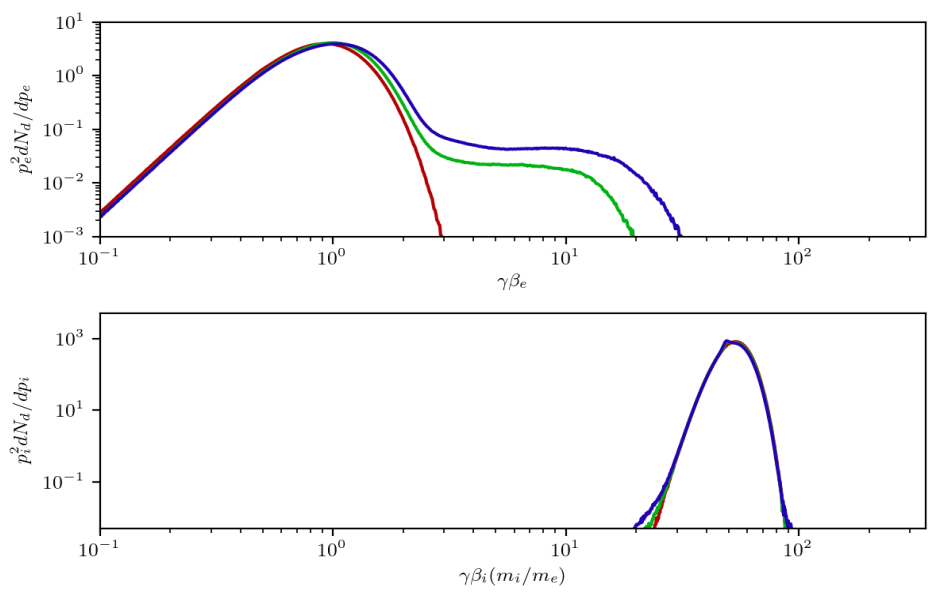

$-45$
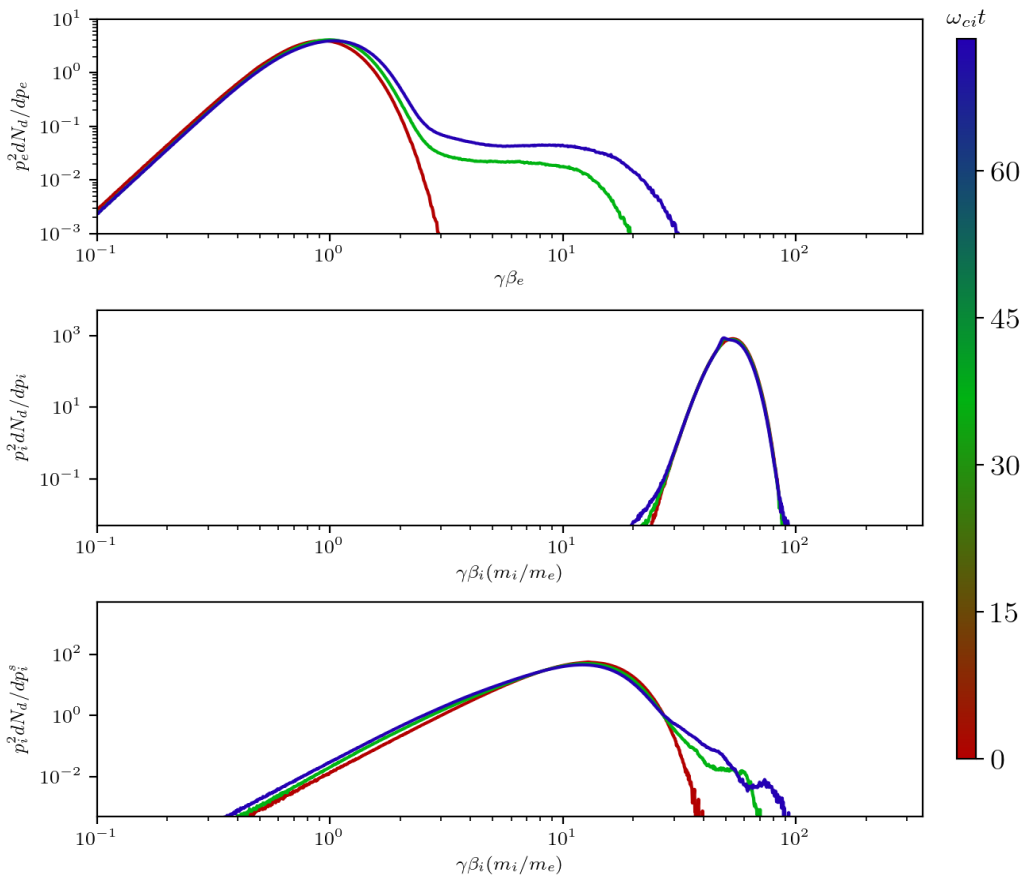

Figure 2: Downstream spectra of electrons (upper panel) and ions (middle panel) in the dHT frame of reference. The bottom panel shows the spectra of ions upon de-boosting from the dHT to shock normal frame and it clearly shows the generation of non-thermal population of ions.
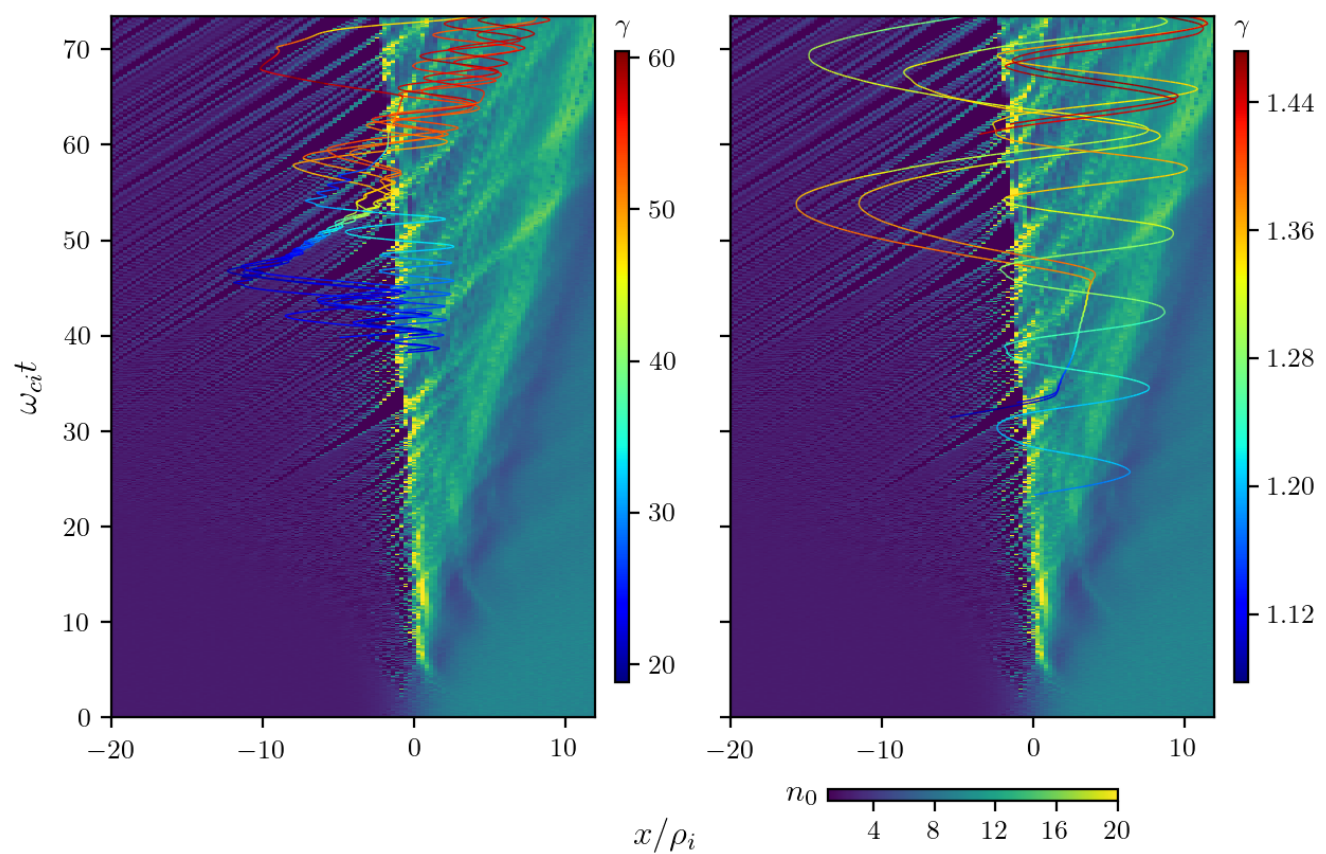

Figure 3: Trajectories of the electrons (first column) and ions (second column) on the downstream side of the shock. Both group of particles show gyrations around the shock. The electron and ion acceleration exhibit several interesting plasma dynamical features such as wave-particle interactions, trapping, shock surfing/reflection, etc. 
acceleration shows build-up with time, though the build-up seems to be slower at later time. One can also notice that ions seem to accelerate after electrons have been accelerated to sufficiently large energies. These accelerated electrons cause significant turbulence in the shock region, facilitating the ion acceleration. Fig. 3 shows the trajectories of five most energetic electrons (left panel) and ions (right panel). One can infer that the electron energization is linked with several microphysical plasma instabilities, electrons gaining energy at the shock foot and also from the interaction with the spiky features arising due to generation of electrostatic and electromagnetic waves $[11,15,16]$. Once electrons have acquired large energies they can resonantly interact with the shock and can subsequently be further accelerated by the DSA or SDA (shock drift acceleration) processes.

\subsection{Test electron trajectories for different mass ratios}

Since the electron and ion acceleration seem to be linked with each other, we study the electron energization process for different mass ratios by initializing two test particles populations in our simulations. These test particles do not take part in generating fields and currents in the simulations but experience the self-consistent plasma fields. They are initialized at varied locations from the shock in the upstream region. Fig.4 shows the spatio-temporal evolution of the energy of these two groups of electrons. One can immediately notice a few salient features: the group of test electrons situated initially further away from the shock (upper row, $\Delta x_{1}=4000 c / \omega_{p e}$ ) gain larger energy compared to the second group initialised closer to the shock (second row, $\Delta x_{2}=1000 c / \omega_{p e}$ ). The test particles for larger mass ratio $\left(m_{i} / m_{e}=256\right.$, first column) gain significantly higher amounts of energy compared to the lower mass ratio cases (second and third columns). Lastly, and most importantly, the electron acceleration is a consequence of several plasma processes at play and which are reflected in the high and low frequency oscillations of these particles. Faster oscillations can be attributed to the electrostatic and electromagnetic plasma instabilities [11, 15, 16]. While the larger period, slow oscillation are either due to the gyration (relativistically corrected) in the magnetic field or the low-plasma frequency modes of oblique electromagnetic Whistler type waves propagating in the upstream direction [15].

\section{Conclusions}

To summarize, we have studied the electron and ion acceleration in a highly oblique nonrelativistic shock by utilizing the shock relaxation method in the de-Hoffmann and Teller (dHT) frame of reference. We have shown the ion acceleration is intricately linked with electron acceleration. We have also shown that the electron injection problem is linked with inertial or space-charge effects. A combination of both electrostatic and electromagnetic instabilities arising due to the reflection of incoming ions at the shock front can provide the requisite amount of electron energization needed for electrons to participate in DSA, ie. their gyroradii exceeding the shock transition width. Our results are relevant for understanding the non-thermal x-ray emission in several astrophysical objects such as Cassiopeia A, Tycho and $\eta$-Carinae [17-19]. 

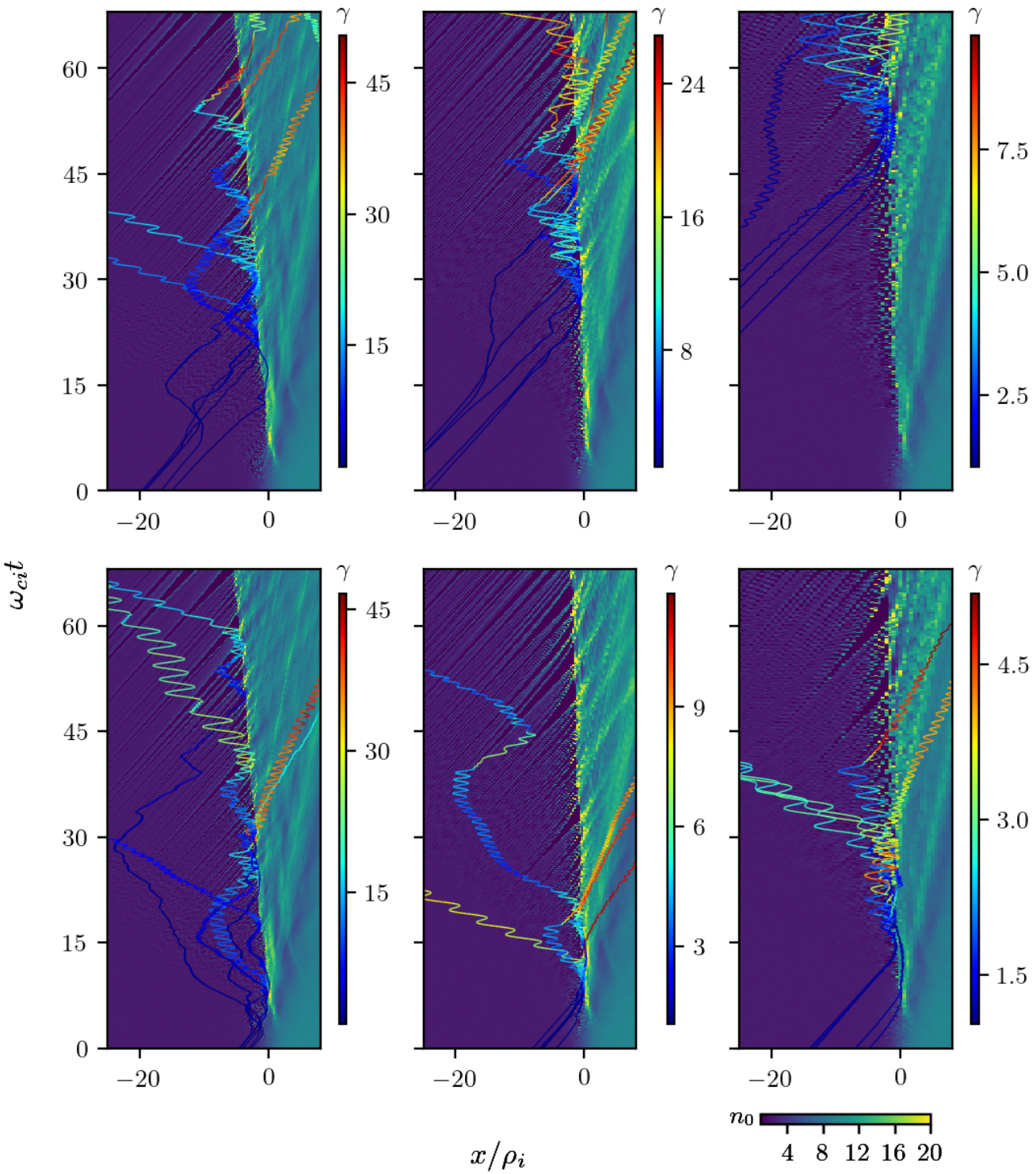

Figure 4: Trajectory of test electrons for different mass ratios. First and second rows represent the two group of test electrons situated further and closer to the shock discontinuity. Columns correspond to these two group of particles for mass ratios $m_{i} / m_{e}=256,100,25$, respectively.

\section{Acknowledgments}

We gratefully acknowledge the use of Raven and Cobra supercomputers and the technical support from Max-Planck Central Data Facility, Munich.

\section{References}

[1] Hinton, J. A. and Hofmann, W., 2009, Ann. Rev Astron. Astrophys., 47, 523

[2] Bell, Anthony Raymond, 2014, Brazilian Jour. Phys., 44, 415 
[3] Blandford, Roger and Eichler, David, 1987, Phys. Reports, 154, 1

[4] Bell, A. R. and Schure, K. M. and Reville, B. and Giacinti, G., 2013, MNRAS, 431, 415

[5] Xu, Rui and Spitkovsky, Anatoly and Caprioli, Damiano, 2020, ApJ, 897, L41

[6] Caprioli, D. and Spitkovsky, A., 2014, ApJ, 783, 91

[7] Derouillat, Julien and Beck, Arnaud and Pérez, F and Vinci, T and Chiaramello, M and Grassi, A and Flé, M and Bouchard, G and Plotnikov, I and Aunai, N and others, 2018, Comp. Phys. Comm., 222, 351

[8] Tidman, D. A. and Krall, N. A., 1971 Shock waves in collisionless plasmas, Wiley Interscience, New York.

[9] Lembège, B. 1990, NATO Advanced Study Institute (ASI) Series C, 305, 81

[10] Bohdan, Artem and Niemiec, Jacek and Pohl, Martin and Matsumoto, Yosuke and Amano, Takanobu and Hoshino, Masahiro, 2019, ApJ, 885, 10

[11] Amano, Takanobu and Hoshino, Masahiro, 2010, Phys. Rev. Lett. 104, 181102

[12] Amano,Takanobu and Hoshino,Masahiro, 2009, Phys. Plasmas, 16, 102901

[13] Leroy, M. M. and Goodrich, C. C. and Winske, D. and Wu, C. S. and Papadopoulos, K., 1981, Geophys. Res. Lett. 8, 1269

[14] Umeda, Takayuki and Kidani, Yoshitaka and Matsukiyo, Shuichi and Yamazaki, Ryo, 2014, Phys. Plasmas, 21, 022102

[15] Muschietti, L. and Lembège, B., 2017, Annales Geophysicae, 35, 1093

[16] Riquelme, Mario A. and Spitkovsky, Anatoly, 2011, ApJ, 733, 63

[17] Patnaude Daniel J. and Fesen, Robert A., 2014, ApJ, 789, 138

[18] Eriksen, Kristoffer A et. al., 2011, ApJ, 728, L28

[19] White, R. and Breuhaus, M. and Konno, R. and Ohm, S. and Reville, B. and Hinton, J. A., 2020, A\&A, 635, A144 